\title{
OUTPUT FEEDBACK SLIDING MODE CONTROL FOR UNCERTAIN DYNAMICAL SYSTEMS
}

\author{
Abdel-Magid M. A. Mohamed \\ Electrical Engineering Department, Aswan Faculty of Engineering, \\ South Valley University, Aswan, Egypt
}

(Received July 2, 2006 Accepted July 20, 2006)

\begin{abstract}
The paper presents a method to design sliding mode control for uncertain dynamical system using only output information. Switching surface is designed through system transformations and pole placement technique. The control law that comprises both equivalent and robust components is investigated. System stability is discussed by using Lyapunov function. Illustrative numerical examples are included to show the applicability and simplicity of the suggested method.
\end{abstract}

\section{INTRODUCTION}

Variable structure control with sliding mode is a robust nonlinear control strategy employing feedback of a discontinuous signal and has been widely applied to various systems [1,2]. A salient feature of this control is that it is completely robust to matched uncertainties that lie in the range space of the input matrix.

In sliding mode control (SMC), there are two basic design problems, the reaching condition and the sliding condition. The former involves choosing a control to move the trajectories of the system onto a preselected manifold within the state space. The latter involves selecting that manifold to give good dynamic characteristics to the closed loop system.

Sliding mode control for linear uncertain systems, under the assumption that all system states are available for measurement, has been studied by using many authors [see, e.g. 1, 2, 3]. The preceding assumption is not usually the case and to overcome this difficulty an estimator may be used to estimate the unmeasured states or use only output variables to implement the control law.

The problem of SMC design for uncertain dynamical systems using output information only has been considered by many authors [4-12]. The advantage of output feedback sliding mode control is using the available outputs of the system and it avoids using an observer to estimate the unmeasurable states.

The purpose of this paper is to investigate the sliding mode control design using only output information for uncertain dynamical system. First, the switching surface is designed by using coordinate transformations and pole placement approach. Switching surface existence condition is obtained. Second, the control vector that comprises both equivalent and robust components is constructed such that the trajectories are driven to and maintained on the sliding surface. System stability under matched uncertainty is guaranteed by using Lyapunov function. Also, numerical examples are included. 


\section{SYSTEM DESCRIPTION}

Consider an uncertain dynamical multivariable system of the form

$$
\begin{aligned}
& \dot{x}=[A+\Delta A(t, x)] x+B[u+w(t, x, u)]+f(t, x, u) \\
& y=C x
\end{aligned}
$$

where $x \in \mathfrak{R}^{n}, u \in \mathfrak{R}^{m}$ and $y \in \mathfrak{R}^{p}$ are the state, the input and the output vectors, respectively, with $m \leq p<n$. $\mathrm{A}, \mathrm{B}$ and $\mathrm{C}$ are constant matrices with appropriate dimensions. $\mathrm{B}$ and $\mathrm{C}$ have full rank, $\operatorname{rank}(\mathrm{CB})=\mathrm{m}$ and $(\mathrm{A}, \mathrm{B})$ is controllable. The term $\Delta A(t, x)$ represents the uncertainty in the linear portion of the plant and $f(t, x, u)$ symbolizes the nonlinearities of the plant while $w(t, x, u)$ can be viewed as the nonlinearities or disturbances at the input. We assume that $\Delta A, f$ and $w$ are bounded. Furthermore, the following matching conditions are assumed to be valid. There exist functions $\alpha($.) and $\gamma($.$) such that$

$$
\begin{gathered}
f(t, x, u)=B \alpha(t, x, u) \\
\Delta A(t, x) x=B \gamma(t, x)
\end{gathered}
$$

Under the matching conditions (2) and (3), system (1a) can be simplified to

$$
\begin{aligned}
& \dot{x}=A x+B[u+\delta(t, x, u)] \\
& y=C x
\end{aligned}
$$

where $\delta(t, x, u)$ represents the lumped uncertainties and/ or nonlinearities:

$$
\delta(t, x, u)=\alpha(t, x, u)+\gamma(t, x)+w(t, x, u)
$$

and assuming that there exist a continuous positive bounded function $\rho($.) such that

$$
\|\delta(t, x, u)\|<\rho(t, u)
$$

where $\|\bullet\|$ indicates the Euclidean norm.

Define the sliding surface as

$$
\sigma=S y
$$

where $S$ is an $m \times p$ matrix to be designed so that the system exhibits desirable dynamical behavior when its trajectories are confined to this sliding surface. The system is in sliding mode when the state lies on the sliding surface after some finite time, i.e.

$$
\sigma=S y=0 \quad t \geq t_{s}
$$

with $t_{s}$ is the time when the sliding mode is reached. The sliding mode control can be accomplished in the following two phases: 
First phase: hyperplane design, that is, given the system (4) and choosing a set of $n-m$ eigenvalues, find the matrix $S$ such that the $n-m$ eigenvalues of system (4) in the sliding mode are precisely those of the chosen set. It should be noted that the reduction of order of (4) to $n-m$ dimensional equivalent system is because the $n$ dimensional state dynamics of (4) must satisfy the $m$ algebraic equation $\sigma=0$. Such constraints reduce the equivalent system from $n$th order system to an $(n-m) t h$ order system [9].

Second phase: Determine a switched control of the form

$$
u_{i}(y)= \begin{cases}u_{i}^{+}(y) & \text { when } \sigma_{i}(y)>0 \\ u_{i}^{-}(y) & \text { when } \sigma_{i}(y)<0\end{cases}
$$

such that the system's trajectory is globally stable to the sliding surface from any point in the output space [2]. The purpose of the control $u$ is to drive the state into the sliding subspace, and thereafter to maintain it there.

\section{SWITCHING SURFACE DESIGN PROCEDURE}

Consider the dynamic model of the system in state space form (4) and use a coordinate transformation

$$
z=R x
$$

where the nonsingular transformation matrix $R$ is chosen so that

$$
R B=\left[\begin{array}{c}
0 \\
B_{2}
\end{array}\right]
$$

where $B_{2} \in \mathfrak{R}^{m \times m}$ is nonsingular. Using (9), system (4) becomes

$$
\begin{aligned}
& \dot{z}=R A R^{-1} z+R B(u+\delta) \\
& y=C R^{-1} z
\end{aligned}
$$

Or, equivalently,

$$
\left[\begin{array}{l}
\dot{z_{1}} \\
\dot{z_{2}}
\end{array}\right]=\left[\begin{array}{ll}
A_{11} & A_{12} \\
A_{21} & A_{22}
\end{array}\right]\left[\begin{array}{l}
z_{1} \\
z_{2}
\end{array}\right]+\left[\begin{array}{c}
0 \\
B_{2}
\end{array}\right](u+\delta)
$$

where $z_{1} \in \mathfrak{R}^{n-m}, z_{2} \in \mathfrak{R}^{m}$, and $A_{11}, A_{12}, A_{21}$ and $A_{22}$ are block matrices with appropriate dimensions. Then form (12), we get

$$
\begin{aligned}
& \dot{z_{1}}=A_{11} z_{1}+A_{12} z_{2} \\
& \dot{z_{2}}=A_{21} z_{2}+A_{22} z_{2}+B_{2}(u+\delta)
\end{aligned}
$$


Subsystem of (13) may be regarded as describing the dynamics of an open-loop control system with state vector $z_{1}$ and control signal $z_{2}$. Noticing that $\left(A_{11}, A_{12}\right)$ is controllable pair of matrices since the pair $(A, B)$ is assumed to be controllable.

The switching surface in the new coordinate is

$$
\sigma=S y=S C x=S C R^{-1} z
$$

Letting

$$
\begin{aligned}
S C R^{-1} & =S\left[\begin{array}{ll}
C_{1} & C_{2}
\end{array}\right] \\
& =D=\left[\begin{array}{ll}
D_{1} & D_{2}
\end{array}\right]
\end{aligned}
$$

where $C_{1} \in \mathfrak{R}^{p \times(n-m)}, C_{2} \in \mathfrak{R}^{p \times m}, D_{1}=S C_{1} \in \mathfrak{R}^{m \times(n-m)}$ and $D_{2}=S C_{2} \in \mathfrak{R}^{m \times m}$ nonsingular matrix. Therefore, on sliding, (15) reduces to

$$
D_{1} z_{1}+D_{2} z_{2}=0
$$

and then

$$
z_{2}=-D_{2}^{-1} D_{1} z_{1}
$$

The dynamic behavior of sliding motion is determined by (13) and (18) that is by viewing $z_{2}$ as the control input to the system (13). The problem of designing an appropriate sliding surface can be regarded as a reduced-order state feedback design problem. So, substituting from (18) into (13), yields

$$
\dot{z_{1}}=\left(A_{11}-A_{12} N\right) z_{1}
$$

where

$$
N=D_{2}^{-1} D_{1}
$$

The matrix $A_{11}-A_{12} N$ is known as the reduced-order equivalent system. So, the design of a stable sliding mode such that $z \rightarrow 0$ as $t \rightarrow \infty$ requires the determination of the gain matrix $N$ such that $A_{11}-A_{12} N$ has $n-m$ left-hand half plane eigenvalues. With taking into consideration that these $n-m$ eigenvalues must contain the invariant zeros of $(A, B, C)$ and it is necessary that these zeros are in open left hand plane [8].

Obviously, the closed loop system dynamics (19) during slide is independent on the control $u$ and depends only on the choice of $S$, which determines the matrix $N$. The convergence of the state vector to the origin is ensured by a suitable choice of the matrix $N$. So, the determination of the matrix $N$, or alternatively, the determination of the matrix $S$, may be achieved without prior knowledge of the control vector $u$. Also, the eigenvalues of the matrix $A_{11}-A_{12} N$ can be placed arbitrarily in the complex plane since the pair $\left(A_{11}, A_{12}\right)$ is controllable by a suitable choice of the matrix $N$. Using (16) and (20), we get

$$
S E=0
$$


where $E=C_{2} N-C_{1}$ known matrix because $N, C_{1}$ and $C_{2}$ are known. Using the generalized matrix inverse [13], the general solution of (21) for $S$ is

$$
S=F\left(I_{p}-E E^{+}\right)
$$

where $E^{+}$is the generalized inverse of $E$ and $F$ is an $m \times p$ arbitrary matrix which must be chosen, if possible, such that $S$ has full rank matrix. Noticing that, to get a full raw rank matrix $S$, the rank of the matrix $E$ must be less than or equals to $p-m$.

\section{CONTROLLER DESIGN}

Now a switched control that drives the system's trajectory to the switching manifold is designed. An approach in variable structure system (VSS) control design is to use the equivalent control, $u_{e q}$ which is the control such that $\dot{\sigma}=0$ for the nominal system, as part of the control vector [4]. Therefore, the control law takes the form

$$
u=u_{e q}+u_{r}
$$

where $u_{r}$ is the robust control, which is switching in nature, developed to guarantee the reaching condition that defined as [2]

$$
\sigma^{T} \dot{\sigma}<0
$$

in the presence of $\delta(t, x, u)$. The equivalent control, $u_{e q}$, will be obtained as follows

$$
\begin{aligned}
\dot{\sigma} & =S \dot{y}=S C \dot{x}=S C R^{-1} \dot{z} \\
& =S C A R^{-1} z+S C B u_{e q}=0
\end{aligned}
$$

Consequently,

$$
u_{e q}=-(S C B)^{-1} S C A R^{-1} z=-(S C B)^{-1} S C A x
$$

Obviously, to implement output feedback, it is required that (26) depends on the output $y$. This can be hold true if there exists a matrix $Q \in \mathfrak{R}^{m \times p}$ such that

$$
S C A=Q C
$$

and then one can write

$$
u_{e q}=-(S C B)^{-1} Q y
$$

and the equivalent control in this case is an explicit output feedback control. Now, the problem of existing the matrix $Q$ is handled. Using the generalized matrix inverse [13], the general solution of (27) for $Q$ is

$$
Q=H C^{+}+M\left(I_{p}-C C^{+}\right)
$$

and the consistency condition is 


$$
H\left(I_{n}-C^{+} C\right)=0
$$

where $H=S C A, C^{+}$is the generalized inverse of $C$ and $\mathrm{M}$ is an $m \times p$ arbitrary matrix. Obviously, because $C$ has full row rank, the second term on the right hand side of (29) will be zero. The condition under which (30) is satisfied can be obtained by letting

$$
W=C A\left(I_{n}-C^{+} C\right)
$$

Then, (30) reduces to

$$
S W=0
$$

Since $S$ has full rank, therefore (32) is satisfied if $\operatorname{rank}(W) \leq(p-m)$, and also if $W=0$. Consequently, the $Q$ matrix exists if $\operatorname{rank}(W) \leq \operatorname{dim} \operatorname{Ker}(S)$ or $W=0$.

An alternative method to get $\mathrm{Q}$ is by assuming the matrix $\mathrm{C}$ has the following form

$$
C=\left[\begin{array}{ll}
0 & T
\end{array}\right]
$$

where $T$ is an $p \times p$ nonsingular matrix. Noticing that the above assumptions of $B$ and $C$ are not restrictions [5,8]. Substituting into (27), yields

$$
S\left[\begin{array}{ll}
0 & T
\end{array}\right]\left[\begin{array}{ll}
- & - \\
A_{11} & A_{12} \\
\bar{A}_{21} & - \\
A_{22}
\end{array}\right]=Q\left[\begin{array}{ll}
0 & T
\end{array}\right]
$$

where $\bar{A}_{11}, \bar{A}_{12}, \bar{A}_{21}$ and $\bar{A}_{22}$ are compatible partitioning of matrix $A$. Equation (34) may be rewritten as

So, if

$$
\left[\begin{array}{ll}
S T \bar{A}_{21} & S T \bar{A}_{22}
\end{array}\right]=\left[\begin{array}{ll}
0 & Q T
\end{array}\right]
$$

Then,

$$
S T \bar{A}_{21}=0
$$

$$
Q=S T \bar{A}_{22} T^{-1}
$$

Now, the robust control may have the following form

$$
u_{r}=-(S C B)^{-1}[\beta \sigma+V \operatorname{sgn}(\sigma)]
$$

where $\beta>0, V=\operatorname{diag}\left[\begin{array}{llll}v_{1} & v_{2} & \ldots \ldots & v_{m}\end{array}\right]$ and $\operatorname{sgn}(\sigma)$ is a vector with components $\operatorname{sgn}\left(\sigma_{i}\right)=1$ if $\sigma_{i}>0$ and $\operatorname{sgn}\left(\sigma_{i}\right)=-1$ if $\sigma_{i}<0$. The components of $V$ is chosen as

$$
v_{i}=\sum_{k=1}^{m}\left|(S C B)_{i k} \| \delta_{k}\right|
$$


where $|\bullet|$ indicate the absolute value of $\bullet$. Therefore, the control vector becomes

$$
u=-(S C B)^{-1} G y-(S C B)^{-1}[\beta \sigma+V \operatorname{sgn}(\sigma)]
$$

\section{Lemma:}

The control vector represented by (40) satisfies the reachability condition (24).

\section{Proof:}

Using (4), (24) and (40), yields

$$
\begin{aligned}
\sigma^{T} \dot{\sigma} & =\sigma^{T}\{(S C A) x-G y-\beta \sigma+(S C B) \delta-V \operatorname{sgn}(\sigma)\} \\
& =\sigma^{T}\{-\beta \sigma+(S C B) \delta-V \operatorname{sgn}(\sigma)\} \\
& =-\beta \sigma^{T} \sigma+\sigma^{T}(S C B) \delta-\sigma^{T} V \operatorname{sgn}(\sigma) \\
& =-\beta\|\sigma\|^{2}+\sum_{i=1}^{m} \sigma_{i} \sum_{k=1}^{m}(S C B)_{i k} \delta_{k}-\sum_{i=1}^{m} v_{i}\left|\sigma_{i}\right| \\
& \leq-\beta\|\sigma\|^{2}+\sum_{i=1}^{m}\left|\sigma_{i}\right| \sum_{k=1}^{m}|(S C B)|_{i k}\left|\delta_{k}\right|-\sum_{i=1}^{m} v_{i}\left|\sigma_{i}\right| \\
& =-\beta\|\sigma\|^{2} \\
& <0
\end{aligned}
$$

This completes the proof.

\section{ILLUSTRATIVE EXAMPLES}

The following examples illustrate the reliability and the practicality of the suggested design procedure.

\section{Example 1:}

Consider a system with the following matrices

$$
A=\left[\begin{array}{ccc}
-1 & 0 & 1 \\
0 & -7 & 5 \\
0 & -3 & 1
\end{array}\right] ; B=\left[\begin{array}{l}
0 \\
0 \\
1
\end{array}\right] ; C=\left[\begin{array}{ccc}
0 & 0 & 1 \\
0 & -1 & 1
\end{array}\right]
$$

Clearly, CB has full column rank and the triple $(\mathrm{A}, \mathrm{B}, \mathrm{C})$ has transmission zero at -1 . So, one arbitrary eigenvalue is chosen at -10 . Using the pole placement method, the matrix $N$ and $E$ are

$$
N=\left[\begin{array}{ll}
0 & 0.6
\end{array}\right], \quad E=\left[\begin{array}{ll}
0 & 0.6 \\
0 & 1.6
\end{array}\right]
$$

$$
\text { Choosing } \quad F=\left[\begin{array}{ll}
9 & 2
\end{array}\right], \quad \text { then } \quad S=\left[\begin{array}{lll}
7.2329 & -2.7123
\end{array}\right] \text {, }
$$

$H=\left[\begin{array}{lll}0.0 & -32.5479 & 18.0822\end{array}\right]$ and $W=\left[0_{2 \times 3}\right]$, then condition (32) is satisfied.

Using (29), yields

$$
Q=\left[\begin{array}{ll}
-14.4658 & 32.5479
\end{array}\right]
$$


It should be noted that condition (36) is also satisfied and the same $Q$ matrix can be obtained by using (37). The time response of outputs $y_{1}$ and $y_{2}$ to an initial condition $\left[\begin{array}{lll}0.9-0.8-0.5\end{array}\right], \beta=30$ and $\delta=0.3+0.1 \sin (10 t)$, is shown in Fig. 1. Also, the corresponding switching function and the control signal are shown in Fig. 2.
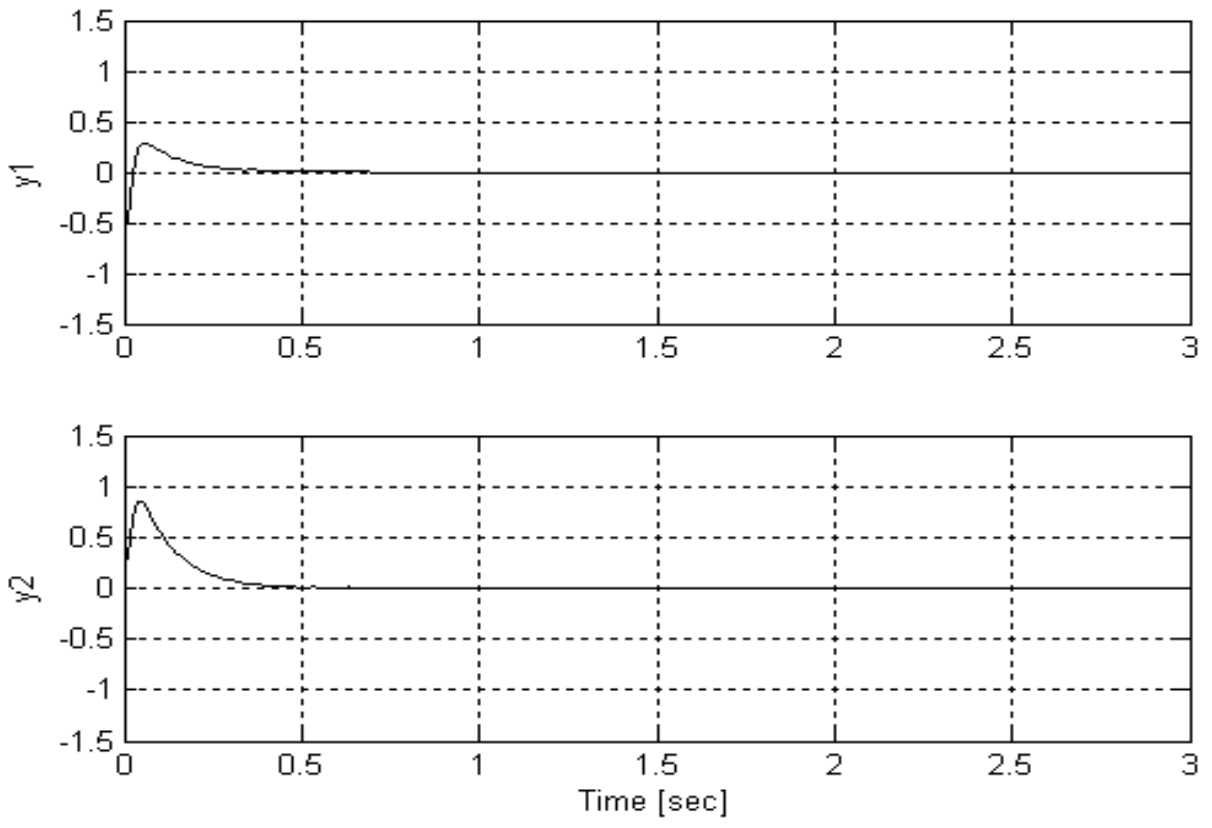

Fig. 1: Output response.
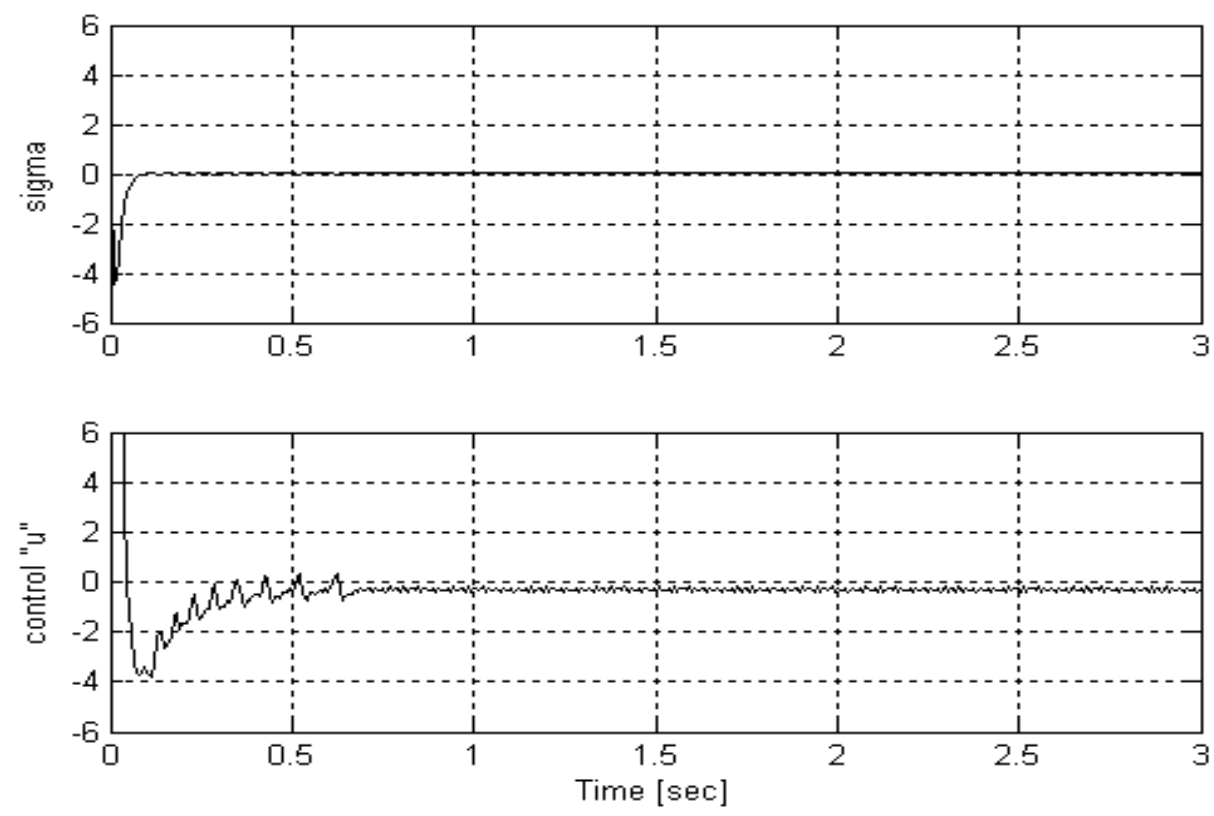

Fig. 2: Switching function and control signal. 


\section{Example 2:}

Consider a system with the following matrices

$$
A=\left[\begin{array}{cccc}
-1.8 & -2.0 & -1.0 & 1.0 \\
0.0 & -2.0 & 0.8 & 0.1 \\
0.0 & 1.0 & -2.0 & 0.0 \\
0.0 & 1.0 & 0.8 & -0.1
\end{array}\right], B=\left[\begin{array}{l}
0.0 \\
0.0 \\
0.0 \\
1.0
\end{array}\right], C=\left[\begin{array}{cccc}
0.0 & 0.0 & 1.0 & 1.0 \\
0.0 & 0.0 & -1.0 & 1.0 \\
0.0 & 1.0 & 0.0 & 0.0
\end{array}\right]
$$

Obviously, $\operatorname{rank}(\mathrm{CB})=1$ and $(\mathrm{A}, \mathrm{B}, \mathrm{C})$ has one transmission zero equals -1.8 . So, choosing two arbitrary eigenvalues at -3 and -4 and using pole placement method, we get

$$
N=\left[\begin{array}{lll}
0.0 & 30.0 & 28.0
\end{array}\right] ; E=\left[\begin{array}{ccc}
0.0 & 30.0 & 27.0 \\
0.0 & 30.0 & 29.0 \\
0.0 & -1.0 & 0.0
\end{array}\right]
$$

Choosing $F=\left[\begin{array}{lll}9.0 & 2.0 & 3.0\end{array}\right]$,we get $S=\left[\begin{array}{llll}2.1708 & -2.0211 & 4.4913\end{array}\right]$, $H=\left[\begin{array}{llll}0.0 & -4.641 & -4.6709 & 0.4342\end{array}\right]$ and $W=\left[0_{3 \times 4}\right]$, and then condition (32) is satisfied. Using (29), yields

$$
Q=\left[\begin{array}{lll}
-2.1184 & 2.5526 & -4.641
\end{array}\right]
$$

Also, it should be noted that condition (36) is satisfied and the same $Q$ matrix can be obtained by using (37). Letting the initial condition be $\left[\begin{array}{llll}0.9 & -0.4 & 0.5 & 0.3\end{array}\right]$, $\delta=0.1+0.5 \sin (10 t)$ and choosing $\beta=15$, the time response of the outputs, $y_{1}, y_{2}$ and $y_{3}$ is shown in Fig. 3. Also, the switching function and the control signal is shown in Fig. 4.
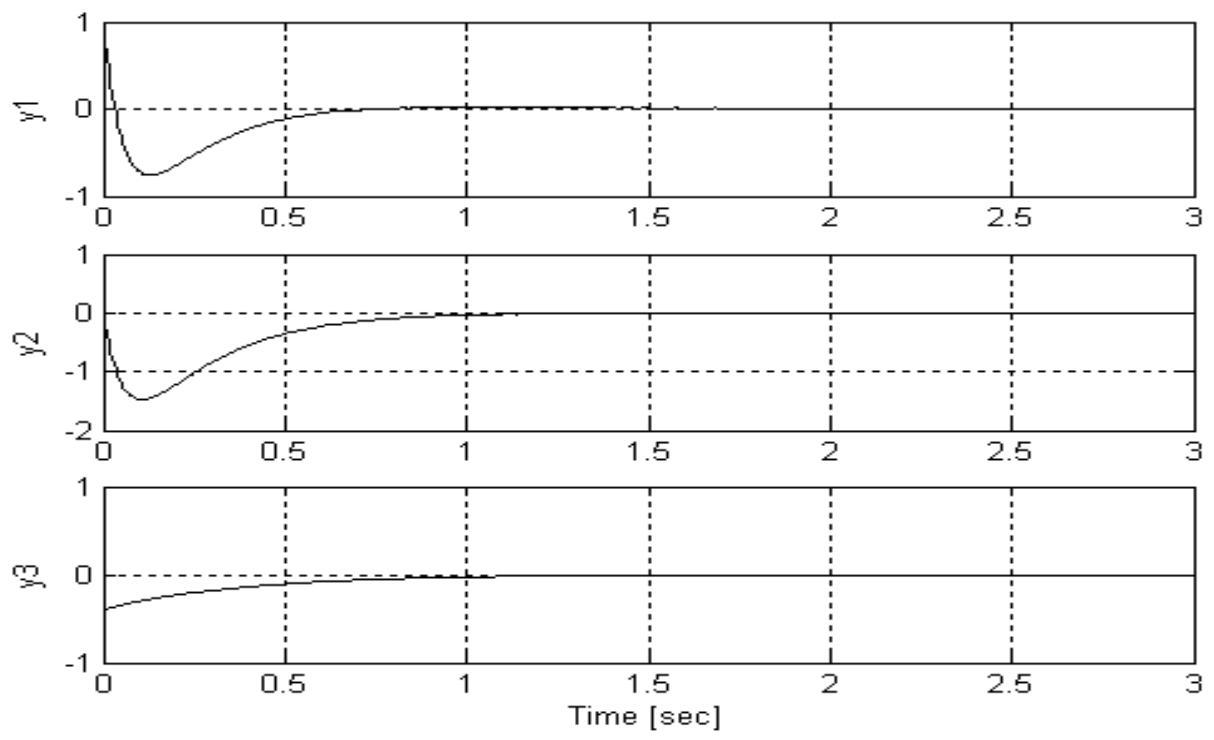

Fig. 3: Output response. 

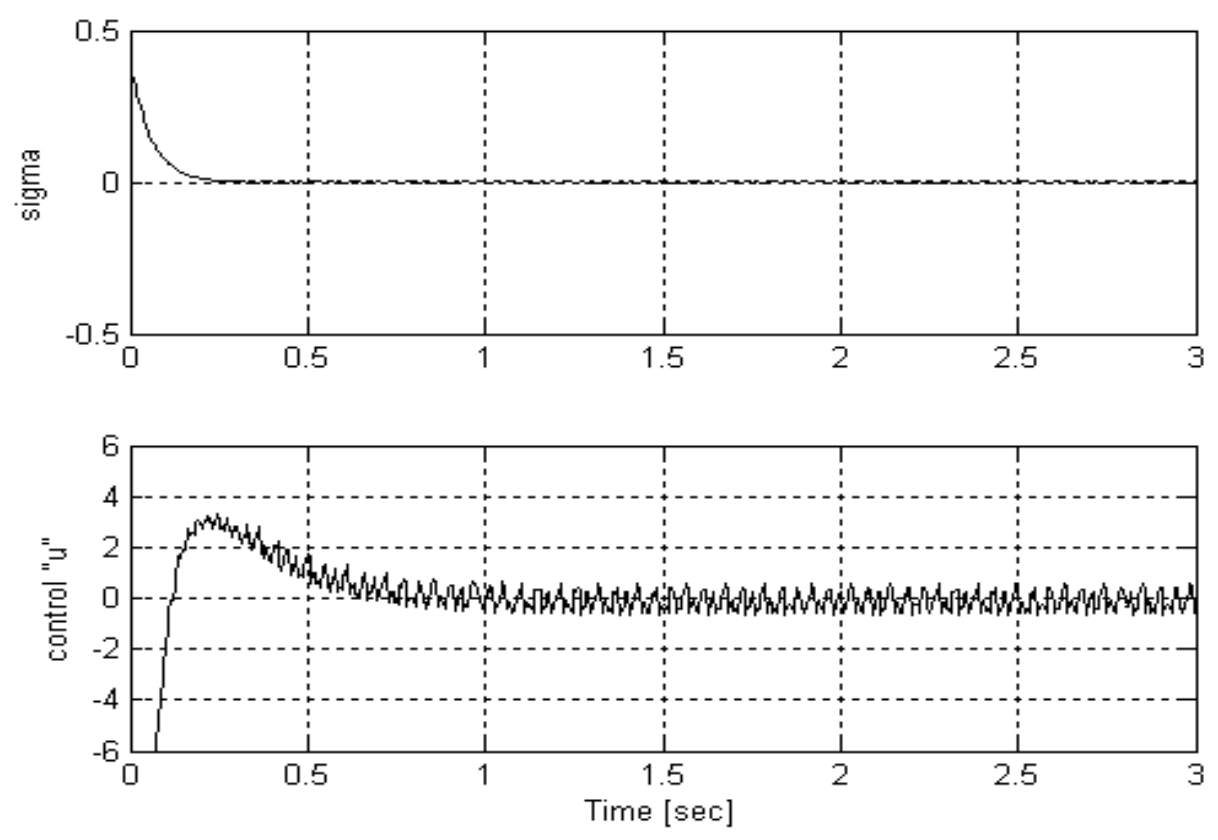

Fig. 4: Switching function and control signal.

\section{CONCLUSIONS}

In this paper a sliding mode controller which requires only output information for a class of uncertain linear system is designed. Switching surface matrix is determined such that the system exhibits desired performance on sliding by using system transformation and pole placemen method. The suggested controller comprises both equivalent and robust components. These components are obtained such that the system stability is guaranteed. Existence conditions for switching surface design and output feedback implementation are given. Illustrative numerical examples were included to show that the suggested method is indeed effective and straightforward.

\section{REFERENCES}

[1] Edwards, C., and Spurgeon, S.K., "Sliding Mode Control: Theory and Applications", Taylor \& Francis Ltd 1998.

[2] Utkin, V.I," "Sliding Modes and Their Application in Variable Structure Systems", Mir Moscow, 1978.

[3] Adamy, J., and Flemming, A., "Soft variable-structure control: a survey", Automatica, 40, pp. 1821-1844, 2004.

[4] El-Khazali, R., and Decarlo, R., " Output feedback variable structure control design”, Automatica, Vol. 31, No. 6, pp 805-816, 1995.

[5] Edwards, C., and Spurgeon, S.K. "On the limitations of some variable structure output feedback controller design”, Automatica, Vol. 36, pp. 743-748, 2000. 
[6] Edwards, C., Spurgeon, S.K., and Hebden, R.G.," On the design of sliding mode output feedback controllers", International Journal of Control, Vol. 76, No. 9/10, pp. 893-905, 2003.

[7] Edwards, C., Akoachere, A., and Spurgeon, S.K., "Sliding-mode output feedback controller design using linear matrix inequalities", IEEE Transactions on Automatic Control, Vol. 46, No. 1, pp. 115-119, 2001.

[8] Edwards, C., and Spurgeon, S.K.," Compensator based output feedback sliding mode controller design", International Journal of Control, Vol. 71, No. 4, pp. 601-614, 1998.

[9] Heck, B.S., Yallapragada, S.V., and Fan, M.K.H.," Numerical methods to design the reaching phase of output feedback variable structure control", Automatica, Vol. 31, No. 2, pp 275-279, 1995.

[10] Ha, Q.P., Trinh, H., Nguyen, H.T., and Tuan, H.D., "Dynamic output feedback sliding mode control using pole placement and linear functional observers", IEEE Transactions on Industrial Electronics, Vol. 50, No. 5, pp. 1030$1037,2003$.

[11] Mohamed, A.-M. M.A., and Mahmoud, M.M.M. " Sliding mode control of uncertain dynamical systems using output information", Journal of Engineering Science, Assiut University, Vol. 33, No.3, pp. 875-886, May 2005.

[12] Xiang, J, Su, H., and Chu, J.," robust sliding mode output feedback control design using ILMI approach", Proceeding of the American Control Conference, June 8-10, USA, pp. 4078-4083, 2005.

[13] Rao, C.R., and Mitra, S.K., "Generalized Inverses of Matrices and Its Applications", (Wiley, New York, 1971).

\section{التحكم الانزلاقى باستخدام متغيرات الخرج للأنظمة الايناميكية اللامحققية}

في هذه المقالة اقترحت طريقة لتصميم التحكم الانز لاقى للأنظمة التي تحوى تغيرات في البارمترات ودخل غير معلوم باستخدام متغيرات الخرج فقط. وتم تصميم السطح

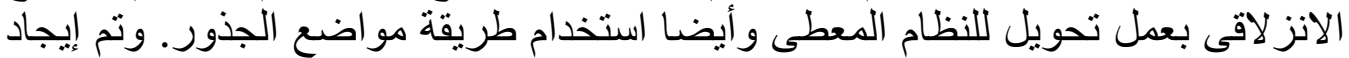

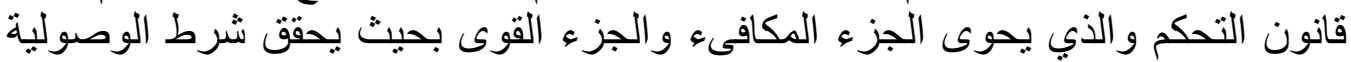
باستخدام دالة ليابنوف. و أوجدت الثروط اللازمة لتصميم سطح الانز لاق وقانون التحكم.

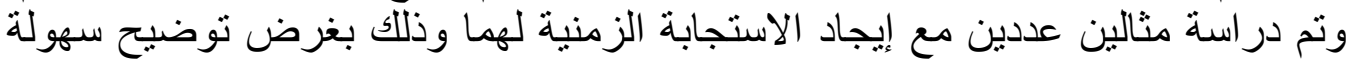
و اعتمادية الطريقة المقترحة. 